\title{
Open-Field Responses of Rats to Odors from Stressed and Nonstressed Predecessors
}

\author{
DAVID A. STEVENS ${ }^{1}$
}

Clark University

and

\author{
E. P. KÖSTER
}

\section{Psychologisch Laboratorium der Rijkuniversiteit, Utrecht}

\begin{abstract}
Male rats were observed on a clean table with extending runways. Paired comparisons were made with clean runways, those on which conspecifics had been stressed, and previously present but not stressed. The subjects stopped more frequently and for a longer duration on runways with predecessors than on clean ones. Stopping was more frequent on runways with stressed predecessors than with nonstressed predecessors. When runways from stressed predecessors were present, there was more frequent urination on the table than when those runways were not used, despite that in the former conditions the subjects spent considerably less time on the table than on the runways.
\end{abstract}

Recent work has demonstrated that odors from surfaces previously occupied by a conspecific have important effects in the behavior of rats. Not only are these findings interesting with regard to their contribution to the general field of animal behavior, but are particularly important in view of the rat's extensive use in studies of learning and motivation, where such odor effects could confound experimentally manipulated variables. For example, the withholding of reinforcement from a rat that had been trained to run a straight alley produces an odor which results in an unconditioned decrement in the running speed of a subsequently tested animal (Wasserman and Jensen, 1969). The reinforcement and withholding of reinforcement from a predecessor have also been shown to be conditions producing odors which can serve as conditioned stimuli for discrimination using runways (Ludvigson and

$\mathbf{1}_{\text {This }}$ research was done at the Psychological Laboratory, State University, Utrecht, supported by a fellowship from the Netherlands Organization for the Advancement of Pure Research (Z. W. O.) and a Fulbright-Hays Travel Award to the first author. 
Sytsma, 1967), and T-mazes (Morrison and Ludvigson, 1970). Male rats prefer odors from sexually receptive females to those from unreceptive females and males (LeMagnen, 1952; Carr, Loeb, and Dissinger, 1965; Carr, Wylie, and Loeb, 1970), and surfaces on which socially submissive animals have had contact are preferred over those from dominant rats (Krames, Carr, and Bergman; 1969).

The results from other studies on the effect of odors from rats of the same sex on exploratory behavior, however, have not been clear-cut. Douglas (1966) found that while male rats avoid their own odor trails in a Y-maze, odor trails from other males have no effect on alley preference, and Halliday (1967) reported that the amount of exploration of a maze by female rats was not affected by previous traversal by another female. Lester (1968) also found that odor trails did not affect the amount of exploration in a Y-maze by females, although sequences of alley entry were less orderly when odor trails were present. One exception to these negative findings is found in an unpublished investigation by Runyon (1954). In that study, Runyon found that rats entered the alley of a Y-maze that had recently contained a shocked rat less frequently than an alley that had not held a rat.

In the context of the negative findings when nonstressed conspecifics have been used, the findings of Runyon suggest that the use of stressed predecessors is a necessary condition to show an influence of conspecific predecessors of the same sex on open-field behavior. In the present study, reactions on an elevated open field to surfaces on which there had been no predecessors, a stressed predecessor, or an unstressed predecessor were observed to test this possibility.

The subjects were 60 male albino rats ( $R$. norvegicus) from the colony maintained at Centraal Instituut voor Voedingsonderzoek (CIVO-TNO), Zeist, ranging in age from 130 to 180 days. Thirty animals served as experimental subjects and 30 served as stimulus animals.

A scale plan of the open-field used for testing is shown in Fig. 1. This was a round glass table from which four runways extended along equidistant axes. The table consisted of a glass plate $70 \mathrm{~cm}$ in diameter placed on top of a sheet of cardboard. This was marked with a line along a diameter which divided the apparatus into halves with symmetrically located runways. Each of the runways was $75 \mathrm{~cm}$ long and $3.5 \mathrm{~cm}$ wide, and also consisted of glass over cardboard. The runways overlapped the table by $3 \mathrm{~cm}$ and a $25 \times 35 \mathrm{~cm}$ glass plate blocked each runway $2 \mathrm{~cm}$ from its distal end. The entire apparatus was supported $50 \mathrm{~cm}$ from the floor by legs or clamps fastened so as to be inaccessible to the subjects. In order to provide a homogeneous surrounding, a screen of white drapes surrounded the apparatus at a distance of $2 \mathrm{~m}$.

The stress- and nonstress- stimulus conditions were obtained by exposing the runways to stressed and nonstressed rats in chambers designed for that purpose. Stressing was done in a box measuring $70 \times 8 \times 20 \mathrm{~cm}$ with wooden 


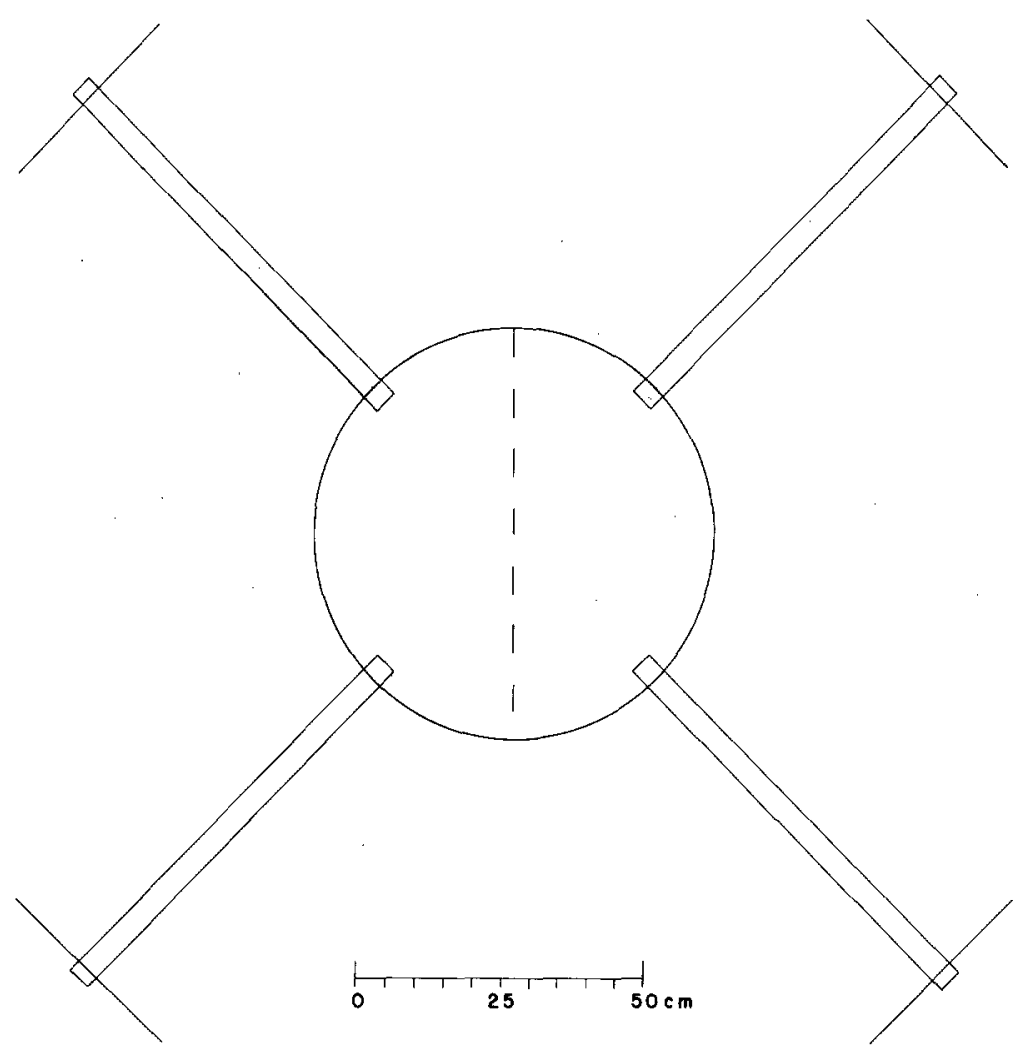

Figure 1. Plan of the open-field test apparatus. Runways on the same side of the midline were of the same stimulus condition.

walls and a glass floor. A gap between one wall and the floor permitted two of the strips of glass used as runways to be inserted on top of the floor. The box was mounted on a swing-type laboratory shaker, set to operate at $150 \mathrm{cpm}$. When activated, intense auditory and visual stimulation was provided by a loudspeaker mounted directly over the box, which produced a $90 \mathrm{db}, 1250$ $\mathrm{Hz}$ tone, and a $200 \mathrm{~W}$ incandescent lamp in a hemispherical reflector mounted $30 \mathrm{~cm}$ above the box. For the nonstress condition, a similar box was used, but this was not mounted on a shaker and there was no loudspeaker or lamp.

The 30 experimental subjects were gentled for $10 \mathrm{~min}$ a day for 7 days, and then randomly assigned to form three groups of ten subjects each. Group S-NS was tested with runways from the stress- and nonstress- stimulus conditions. Group S-C was tested with runways from the stress-stimulus condition and clean runways, and Group NS-C animals were tested with runways from the nonstress-stimulus condition and clean runways. 
Ten rats served as stimulus animals for the stress stimulus condition. These were not handled except at weaning and for occasional cage cleaning. For this stimulus condition, clean runway strips were placed over the floor of the stress box. One of the stress animals was placed in the box and the shaker, loudspeaker and lamp were turned on for $3 \mathrm{~min}$. When necessary, a clean piece of glass was held over the apparatus to prevent escape by the animal. At the end of the stressing period the animal was removed, and after fecal boluses and urine were shaken off the two strips were affixed to the table, adjacent, rather than opposite, to one another. Each stress-stimulus animal was used twice, with at least $24 \mathrm{hr}$ separating the stress sessions.

Twenty rats were used as stimulus animals for the nonstress-stimulus condition. These were handled for 3 weeks prior to the experiment, and were allowed to individually explore the interior of the nonstress-stimulus chamber for $5 \mathrm{~min}$ on each of 2 days prior to testing. The procedure for the nonstress-stimulus condition was identical to that used to obtain the stress stimulus, except that no shaking, or extra auditory or visual stimulation was employed. This treatment was given in a different room than the other so that both treatments could be given simultaneously. Experimenters carried the runways to the table by grasping them with clean paper, and took care to touch only the last $2 \mathrm{~cm}$ which would extend beyond the vertical barrier, or the underside, so that the portion of the runway to which the subjects had access during testing was not contaminated.

Each experimental subject was tested once. Testing consisted of placing a subject at the center of the table with its body oriented along the midline of the apparatus, facing away from the experimenter. The experimenter then withdrew to his observation post and subject was observed for 9 min. Between tests, the glass floor of the table was carefully scrubbed and rinsed with water. The order in which individuals from the three experimental groups were tested was counterbalanced, as was the location of the runways for the three stimulus conditions with respect to the position of the observers behind the screen. The number of times an animal stopped (no movement of four feet), the duration of each stop, and the location of each stop were recorded. Stops were used as the criterial behavior since observations indicated that in an open-field situation, sniffing is usually accompanied by stopping.

Table 1 shows the mean number of times the subjects stopped moving, and the mean total duration of those stops on the runways and associated sides of the table. In the two conditions in which stressed predecessors were used (Groups S-NS and S-C), the subjects stopped more frequently and longer on the runways with stressed predecessors than on those with nonstressed predecessors, or on clean runways. The animals tested with runways from nonstressed predecessors and clean runways (Group NS-C) stopped more frequently and for longer durations on the former than on the latter. Differences within groups were analyzed by $t$ tests; all differences were statistically significant except for the duration scores for Group S-NS. 
Within-group differences in frequency and duration of stops on the table were, with one exception, in the same direction as found on the runways, but in no case were they statistically significant.

TABLE 1

Mean Frequency and Mean Total Duration of Stops on Runways and Table

\begin{tabular}{|c|c|c|c|c|c|c|}
\hline & \multicolumn{3}{|c|}{ Frequency } & \multicolumn{3}{|c|}{ Duration (sec) } \\
\hline \multicolumn{7}{|c|}{ Group S-NS } \\
\hline & $S$ & NS & $t^{\mathrm{a}}$ & $S$ & NS & $t$ \\
\hline Runways & 17.0 & 9.3 & $2.83^{b}$ & 204.1 & 101.9 & 1.54 \\
\hline Table & 10.1 & 7.1 & 1.26 & 77.0 & 54.0 & 0.63 \\
\hline \multicolumn{7}{|c|}{ Group $S-C$} \\
\hline & $S$ & $\mathrm{C}$ & $t$ & $S$ & $\mathrm{C}$ & $t$ \\
\hline Runways & 13.6 & 7.0 & $2.86^{b}$ & 243.5 & 36.5 & $3.91^{\mathrm{b}}$ \\
\hline Table & 9.3 & 6.7 & 1.47 & 73.4 & 84.1 & 0.23 \\
\hline \multicolumn{7}{|c|}{ Group NS--C } \\
\hline & NS & $\mathrm{C}$ & $t$ & NS & $\mathrm{C}$ & $t$ \\
\hline Runways & 13.8 & 4.3 & $4.56^{\mathrm{b}}$ & 282.6 & 28.9 & $4.62^{b}$ \\
\hline Table & 9.0 & 7.4 & 1.40 & 108.6 & 51.6 & 1.83 \\
\hline
\end{tabular}

$a_{d f}=9$ for all tests.

$\mathrm{b}_{P}<.02$, two-tailed test.

Whether or not urination occurred was related to the nature of the test conditions. Of the 20 animals tested in the two conditions in which runways treated by stressed predecessors were used (Groups S-NS and S-C), 19 urinated, but of the ten animals in the remaining condition (Group NS-C), only six urinated. Fisher's Exact Test indicated that this difference in frequency of animals urinating was statistically significant $(P=.031)$. Almost all urination took place on the table; only two of the 25 animals which urinated did so on the runways. Defecation was observed by six animals in Group S-NS, three in Group S-C and five in Group NS-C. This difference was not statistically significant. All defecation took place on the table. 
The present results clearly show that previous contact of a surface by a male rat affects the behavior of another male rat when on that surface. Regardless of whether or not predecessors were stressed, the subjects in the present study stopped more frequently and for longer duration on surfaces on which predecessors had contact. While the use of stressed predecessors is not a necessary condition to show an effect, the finding of a greater frequency of stopping on stressed-predecessor-treated surfaces and of more frequent urination when those surfaces were used than when they were not indicates an unconditioned discrimination between them and surfaces treated by nonstressed animals.

Valenta and Rigby (1968) were able to establish a conditioned discrimination in rats between odors from stressed and nonstressed conspecifics. On the basis of this finding Ralls (1971) stated that the existence of an alarm pheromone in the rat was probable. The results of the present experiment can be taken as support for Ralls' hypothesis only if the stopping and urination can be interpreted as alarm reactions. However, this interpretation is difficult to make. First, rats in Group NS-C stopped about as frequently on runways treated by nonstressed animals as the rats in Group S-C stopped on runways treated by stressed animals. Thus, the use of stressed predecessors was not necessary to produce frequent stopping. Second, if the stops were an alarm reaction (i.e., emotional freezing) they should be accompanied by urination and defecation. But, there was no more defecation found in the conditions which employed runways from stressed animals than that which did not. Furthermore, all defecation and almost all urination occurred on the table, not on the runways. This occurred despite the fact that the runways were the only surfaces on which predecessors had contact and where significant differences in stopping were found, and that the subjects spent far more time on the runways than on the table. Thus, while more frequent urination was found when runways treated by stressed predecessors were present, the location of these responses suggests that they were not a released alarm reaction. The fact that the frequency of animals urinating depended upon the use of stressed predecessors, together with the fact that the location of the urination was different from the location where the stimulus was found suggests that the subjects were marking. This identification, however, must remain speculative until further work shows that such urine has a signaling function. Nevertheless, while stressing may not produce an alarm pheromone, it seems likely that stressed rats do produce an odor with pheromonal qualities.

From the present data it cannot be established if the behavioral differences found were due to qualitative differences, or quantitative differences in the predecessors' odors. It is possible that the stressed predecessors' odors were more intense than the nonstressed predecessors' and the differences in behavior observed on the runways simply reflect differences in 
adaptation and/or habituation to an otherwise similar odor. On the other hand, the finding that urination on the table was more frequent when runways treated by stressed predecessors were present suggests that qualitatively different odors were produced by the different treatments given the predecessors. Further analyses, either chemical or behavioral, are needed to answer this question.

The present results were in the opposite direction of those reported by Runyon (1954), who found that rats avoid a location where a predecessor was stressed. The explanation for this might lie in the methods used to produce stress. Runyon used painful electric shock while in the present study stress was obtained by given proprioceptive, visual and auditory stimulation to animals which had a minimum of previous handling. This treatment, while aversive, did not elicit squealing and was probably below the threshold of pain. This difference in intensity of stimulation might account for the differences found. Another possibility is that electric shock can produce artifactual odors such as ozone, or from the burning of skin or hair, which could be aversive. Artifactual odors were not possible with the present method.

\section{REFERENCES}

Carr, W. J., Loeb, L. S., and Dissinger, M. L. (1965). Responses of rats to sex odors. $J$. Comp. Physiol. Psychol. 59, 370-377.

Carr, W. J., Wylie, N. R., and Loeb. L. S. (1970). Responses of adult and immature rats to sex odors. J. Comp. Physiol. Psychol. 72, 51-59.

Douglas, R. J. (1966). Cues for spontaneous alternation. J. Comp. Physiol. Psychol. 62, 171-183.

Halliday, M. S. (1967). The influence of olfactory cues on exploratory behavior. Psychon. Sci. 9, 595-596.

Krames, L., Carr, W. J., and Bergman, B. (1969). A pheromone associated with social dominance among male rats. Psychon. Sci. 16, 11-12.

LeMagnen, J. (1952). Les Phenomenes olfacto-sexuels chez le rat blanc. Arch. Sci. Physiol. 6, 295-332.

Lester, D. (1968). Effects of olfactory stimuli on Y-maze exploration of rats. Psychon. Sci. 12,97 .

Ludvigson, H. W., and Sytsma, D. (1967). The sweet smell of success: Apparent double alternation in the rat. Psychon. Sci. 9, 283-284.

Morrison, R. R., and Ludvigson, H. W. (1970). Discrimination by rats of conspecific odors of reward and nonreward. Science 167, 904-905.

Ralls, K. (1971). Mammalian scent marking. Science 171, 443-449.

Runyon, R. P. (1954). The role in avoidance of odors produced by stressed rats. Unpublished doctoral dissertation, Yale University.

Valenta, J. G., and Rigby, M. K. (1968). Discrimination of the odor of stressed rats. Science 161, 599-601.

Wasserman, E. A., and Jensen, D. D. (1969). Olfactory stimuli and the "pseudoextinction" effect. Science 166, 1307-1309. 